\title{
Modeling Topics In The Alternative Uses Task
}

\author{
Richard W Hass ${ }^{1}[0000-0002-9545-4800]$ \\ Thomas Jefferson University, Philadelphia PA USA \\ hassr@philau.edu
}

\begin{abstract}
What is the representation of knowledge underlying human responses to alternative uses test items? This short paper describes an application of Latent Dirichlet Allocation (LDA)-also known as topic modeling-to solve this problem of knowledge representation. For this small application, a document was defined as the set of responses given by a single participant to the alternative uses test "brick" prompt. This was chosen instead of single responses as the document unit, as single responses to alternative uses items are rather short, and LDA assumes that documents are probabilistic mixtures of topics. The approach explored in this paper used LDA with Gibbs sampling, with the primary goal of model selection. The $\log$ likelihood of the data $(\log P(\mathbf{w} \mid \mathbf{T}))$ was computed as to topics varied from 5 to 100 . Results showed that the log likelihood increased to a peak at 15 topics and then steadily declined up to 100 topics. In the 15-topic model the most frequently appearing topic was that which gave the highest probability to the terms build, house, step, and smash. Documents best represented by that topic assignment were, on average, more similar to the dictionary definition of a brick based on vector cosines computed with Latent Semantic Analysis. Additional implications for using the topic model as a knowledge base for cognitive systems, and also as a tool for quantifying "flexibility"-the number of categories present in alternative uses response arrays-will also be discussed.
\end{abstract}

Keywords: Creativity Tests · Creative Cognition · Structured Representations

\section{Introduction}

Modeling concepts and knowledge structures is daunting from both a psychological and computational point of view. Recent advances in information retrieval and natural language processing have been adopted by cognitive scientists for use in understanding the conceptual foundations of linguistic information. Two approaches are particularly important-latent semantic analysis (LSA) and latent Dirichlet allocation (LDA)-both of which are "bag of words" methods used in information retrieval to gleam semantic information from text. While both have limitations, each algorithm has shown promise for representing the structure of semantic memory $[5,10]$. 
This paper describes the use of LDA as a model of the semantics underlying the generation of alternative and creative uses for objects. The paper is organized as follows. First, a brief overview of creative idea generation methodology is given, including some descriptions of current cognitive models of the process. Next, LDA is described in the context of "topic modeling", and existing applications of the algorithm are discussed. Then, details of an LDA (with Gibbs sampling) topic model of creative uses for bricks will be described, along with validation efforts. Key to validation was the comparison of the topic assignments for documents in the current corpus and LSA cosine similarity between each document-a collection of one participants brick uses-and a dictionary definition of brick previously used by $[6,7]$ as a representation of the brick concept in LSA space. The paper ends with a discussion of limitations and future directions.

\subsection{Creative idea generation}

Idea generation plays a central role in creativity, and represents one of the most common ways of measuring creativity in psychological research. When measured, it is usually referred to as "divergent thinking" an extension of a term coined by [8] to describe the production of novelty by the human intellect across a variety of contexts, including problem solving. This paper focuses on one particular divergent thinking task: the Alternative Uses Task (AUT). One of the most common objects used as a prompt for the AUT is the brick. To obtain data in experimental settings, participants are usually given some interval of time (e.g., 3 minutes) to "think of creative, unusual, uncommon, and/or novel" uses of a brick. In some cases, additional instructions that prioritize "quality or creativity" over sheer volume of responses (fluency) are provided to participants for various reasons not central to the goal of this analysis. People typically are able to generate between 4 and 8 alternative uses for objects such as a brick in 3 minutes time, the most common time interval used in the research.

There currently no accepted "theory" of divergent thinking, but in the last decade, research has increasingly pointed to relations between idea generation and fluid intelligence-the human ability to quickly and adaptively switch strategies during problem solving $[1,6,9,12]$-along with further support of the notion that creative idea generation is rooted activating and retrieving remotely associated knowledge [7,9]. Importantly, the dynamic connectivity among brain networks supporting attention, executive functions and memory retrieval has been implicated as an important correlate of idea generation performance [2]. Taken together, generating simple, novel ideas, seems to rest on exploring remote connections among memory units, and potentially exploiting newly found connections.

One clue that divergent thinking is rooted in semantic memory retrieval is that the relation between the rate of production and time elapsed during divergent thinking mirrors that which is also found in simple semantic recall tasks [7]. What is important about that observation, is that it is consistent with the type of random memory processes described in a number of computational models of recall $[14,19]$. That is, the divergent thinking process involves a kind of 
random memory search that is similar to the search for semantic associates in normal memory recall. However, the difference between the two search processes is that in divergent thinking, the search does not seem to exploit local semantic relations in the same way as normal recall. For example, in recalling animals, people usually produce examples in semantically related clusters (e.g., farm animals, pets, etc.). Hass [7] showed that this is not the case for divergent thinking. Instead, divergent thinking responses are emitted more slowly, with adjacent responses often having low semantic similarity. There are two equally plausible explanations for this phenomenon. First, it is likely that divergent thinking does not simply involve searching for known instances of object uses in memory and then emission of those uses, but rather that it involves an extra generation step, whereby inferences are made about the properties of the objects that might afford different, non-standard uses [13]. Alternatively, it may be that the search process active during divergent thinking operates according to an algorithm that restricts the semantic similarity between adjacent responses, and that the production of novelty is a function of the search process. The currently presented model of divergent thinking responses cannot adjudicate between these two explanations, but can provide a kind of model of the feature space that the process might operate over. As discussed in the next section, the modeling of semantic information with latent Dirichlet allocation results in a set of "topics", which are collections of terms connected to the content of language through a generative probability model. The goal of the analysis was to probe the structure of a topic model of divergent thinking responses in order to shed more light on the structure of the underlying memory search process.

\subsection{Topic modeling}

Topic modeling with latent Dirichlet allocation (LDA) is a probabilistic method for representing the meaning of a unit of natural language [3], but holds promise as a model of human semantic memory [5]. LDA is more easily understood when contrasted with another representation of meaning, latent semantic analysis (LSA). Like LDA, LSA is a bag of words approach to processing the semantic content of a linguistic document of arbitrary size. The mathematical and computational details of the singular value decomposition algorithm at the heart of LSA are available elsewhere [10, e.g.]. Briefly, the algorithm seeks to find a set of latent variables that can reduce the dimensionality of the term-document matrix representing a corpus. The matrix decomposition that achieves the result is similar to factor analysis, and the result that the meaning of a word or phrase is encoded as a coordinate in this latent semantic space. While comparisons between words in LSA space provide a good approximation to human similarity judgements [10], the plausibility of singular value decomposition as a mental process was noted as a limitation of LSA from the start. Griffiths, Steyvers, and Tennenbaum [5] noted other limitations of the LSA spatial representation, most notably, the difficulty in resolving semantic ambiguities like polysemy. Their paper compared LDA (obtained using Gibbs Sampling) with LSA using the same corpus, and found that the LDA topic model performed as well if not better than 
LSA in predicting the behavior of humans in a variety of semantic memory tasks. More importantly, they argued that unlike LSA, in which similarity is modeled as a spatial relationship, similarity in topic models is feature-based. Feature-based theories of similarity are generally seen as more plausible for humans [17], and may be more appropriate for modeling the process of creative thinking during the AUT task [13]. Thus, LDA and topic modeling has the potential to both aid in the understanding of human knowledge used in AUT tasks, and also to model that knowledge for use in a cognitive system.

Brief summary of LDA Modeling topics with LDA involves estimating parameters in a hierarchical generative probability model. There are several approaches to estimating the parameters, and this paper used Gibbs Sampling [4]. Grun and Hornik [16] provided an excellent overview of the derivation of all approaches, and only a brief summary will be given here. An LDA model describes a generative process for a particular document $d_{i}=w_{1}, \cdots, w_{j}$ from a corpus $C$ consisting of $N$ words belonging to a vocabulary $V$. Grun and Hornik describe a three-step generative process consisting of:

1. Determine the term distribution $\phi$ for each topic as $\phi \sim \operatorname{Dirichlet}(\beta)$

2. Determine the topic distribution $\theta$ for each document as $\theta \sim \operatorname{Dirichlet}(\alpha)$

3. For each of the words $w_{j}$, chose a topic $t_{i} \sim \operatorname{Multinomial}(\theta)$, and choose a word from another multinomial distribution conditioned on the chosen topic $t_{i}: p\left(w_{j} \mid t_{i}, \phi\right)$.

In the final step, $\phi$ is the term distribution over topics, which provides the probability of a word occurring in a particular topic.

The estimation of $\phi$ and $\theta$ were accomplished using the Gibbs sampling procedure described by [4] which, as in the above generative model, sets Dirichlet priors on the term and topic distributions, by fixing $\beta$ and $\alpha$, respectively. The procedure results in an estimate of the posterior distribution of of topic assignments for $\mathrm{D}$ documents given the words $p(t \mid w)$ by drawing samples from a modified version of the proceeding distribution consisting of topic assignments omitting particular words. Final estimates for the multinomial distributions $\phi$ and $\theta$ are then obtained using the word and topic assignments and hyperparameters $(\beta$ and $\alpha)$.

One caveat to the procedure is that the number of topics must be fixed, a priori, in order for estimation. In the current analysis, one question is thus, how many topics must be specified a priori to arrive at an adequate representation of the semantics of object uses? Following [4] the current analysis fixed $\beta$ and $\alpha$ and varied the number of topics. A final topic model was chosen by maximizing the $\log$ likelihood of the term distribution $(\log P(\mathbf{w} \mid \mathbf{T}))$ as topics varied. This is an empirical step for model validation, however, it is assumed that the mind achieves a set of topics via some process of statistical learning, and that this number is not varied when performing a similarity judgment, or utilizing semantic memory to produce novel responses. 


\section{Data and Model description}

\subsection{Data preparation}

The data used for this analysis were culled from several human subjects experiments using standard divergent thinking protocols. In each case, participants were given 3 minutes to generate creative, alternative uses for a brick. These responses were typed into computer terminals, and compiled into spreadsheets for analysis. The entire dataset, along with all of the code for performing the preparation and analysis are available here: https://osf.io/fv7xb/. A corpus was formed by setting document size to be the set of responses given by a single person in the context of an AUT-brick task. For example, document 1 was from a participant who gave 9 responses (separated by commas here): "doorstop, weapon, gravity demonstration, bookend, landmark, pressing flowers, stepping stone, lifting, flyswatter." As can be seen, single responses are rather short and it was thought that treating a full set of responses from one participant as a single document would yield a better model of topics overall. Tools from the $t m$ [15] R package were used to format the data. Along with removing stopwords (see [15]), punctuation was removed, and any extraneous white space. Term-frequency inverse-document-frequency weighting was used to further remove low frequency terms. The resulting corpus consisted of 363 documents, 988 terms, and 4916 tokens. Because each document is tied to a single experimental participant, this corpus represents data from 363 distinct people.

Estimation with Gibbs Sampling Estimation was performed using the topicmodels $\mathrm{R}$ package [16]. As discussed, estimation of the posterior distribution of topic assignments given the words was accomplished with Gibbs sampling (1000 iterations, after a burn-in of 1000, and keeping every 100th sample), with $\beta$ set to $\frac{50}{T=k}$, where $\mathrm{k}$ varied from 5 to 100 topics across models in units of 5 , and fixing $\alpha$ at .01. For each of the 20 different models (for topics ranging from 5 to 100) $\log P(\mathbf{w} \mid \mathbf{T})$ was extracted from the returned results. For the best fitting (highest log-likelihood) topic model, further tools from the topicmodels package were used to produce the term distributions for each topic.

\section{Results and validation}

\subsection{Model Comparison}

The first empirical question addressed by this analysis was that of the number of topics necessary to model the semantic content of the corpus. Figure 1 illustrates $\log P(\mathbf{w} \mid \mathbf{T})$ for models with topics varying from 5 to 100 . As can be seen, the log likelihood was maximized when 15 was specified as the number of topics. Log likelihood values then declined monotonically as topics varied from 20 to 100. This result suggests that 15 topics can accurately capture the semantic content encoded in participants' brick responses. However, this result does not fully validate the 15 topic model. Validation of this model is discussed next. 


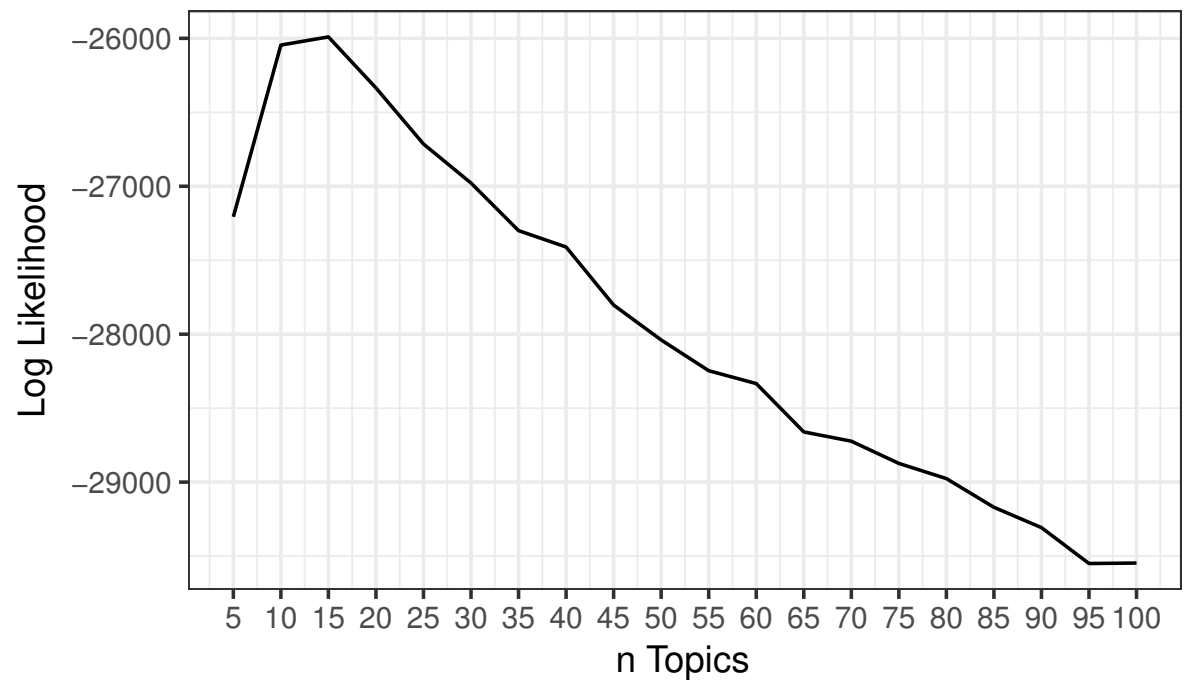

Fig. 1. Log likelihood values for models with topics varying between 5 and 100

Model validation Two related analyses were performed in order to determine whether the 15 topic model adequately represented the semantic content of the AUT-brick responses. First, it is well known that people tend to give many responses to alternative uses tasks that are similar to the conventional use for the object (in this case a brick). So one way to validate the 15 topic model is to examine whether topics that correspond to a conventional brick definition appear often in the corpus. Table 1 lists the five most probable terms per topic along with the frequency with which a document in the model was "assigned" to that topic. Topic assignments represent the highest probability topic for a particular document in the corpus, though the model assumes that documents are mixtures of topics. Interestingly, the most frequent topic assignment was that of topic 2, which gives high probability to words like weapon, lift, step, stone, and break. Terms that correspond to the "usual" use of a brick as building material are given high probability under Topic 5 , which was also the 3rd most frequent topic assignment. This result suggests that the model qualitatively seems to capture elements of common responses in the corpus.

Simply examining the words from a particular topic is perhaps good qualitative evidence of a good fitting model, but for further validation, LSA was used to compare each document to a reference document, the dictionary definition of a brick. Each of the 363 documents was compared to a composite dictionary definition of a brick in LSA the TASA 300-factor space provided by the tools at the UC Boulder website (lsa.colorado.edu). The definition is based on that given by Merriam-Webster (http://www.merriam-webster.com/dictionary/brick) and is as follows: "a small, hard block of baked clay that is used to construct houses and sometimes to make streets and paths". Note that the Merriam-Webster def- 
Table 1. Summary of the output and validation of the 15-topic model. Five most probable terms (stemmed) for each of the 15 topics are in column 2. Column 3 gives the number of documents $\left(N_{\text {total }}=363\right)$ with highest probability under each topic. Column 4 is the Mean of the cosine similarities (LSA space) between the dictionary definition of "brick" and each document assigned high probability across the 15 topics.

\begin{tabular}{|c|c|c|c|}
\hline \multicolumn{2}{|c|}{ Topic Top 5 Terms } & \multicolumn{2}{|c|}{$\begin{array}{c}\text { Mean } \\
\text { Freq. similarity }\end{array}$} \\
\hline 1 & window decor stop weight break & 33 & .130 \\
\hline 2 & weapon lift step stone break & 49 & .139 \\
\hline 3 & car throw break paint wall & 22 & .142 \\
\hline 4 & use can play paper like & 43 & .175 \\
\hline 5 & build hous paper stopper road & 41 & .256 \\
\hline 6 & make art book stand write & 22 & .114 \\
\hline 7 & weight someon build smash roll & 16 & .105 \\
\hline 8 & weight thing block step door & 19 & .109 \\
\hline 9 & paperweight doorstop place step water & 24 & .098 \\
\hline 10 & door hammer garden sidewalk build & 19 & .090 \\
\hline 11 & brick door tool break exercis & 15 & .157 \\
\hline 12 & tabl flower bed pot shoe & 18 & .088 \\
\hline 13 & hold put pencil holder bridg & 17 & .079 \\
\hline 14 & someth open break prop stopper & 21 & .093 \\
\hline 15 & hole paperweight chair holder fire & 4 & .130 \\
\hline
\end{tabular}

inition uses the word "build" rather than "construct." The change was made to the current definition to avoid large similarity values for documents containing the word "build." The mean cosine similarity was then calculated across all documents assigned to each of the 15 topics, and those values appear in Table 1 (column 4).

Two interesting results emerged from the LSA analysis. First, there was a moderate correlation $(r=.50, p=.03)$ between frequency of topic assignments (column 3) and mean cosine similarity (column 4) per topic. This is sensible if one considers low frequency topics to reflect uncommon responses to the AUTbrick task. Hass [6] showed that cosine similarity and creativity are negatively correlated, so the fact that lower-frequency topics were also marked by smaller cosine similarities to a brick suggests that those topics are more remote concepts, which may in turn be more creative. However, further analysis-using actual creativity ratings of the current corpus-is necessary to confirm this assertion.

The more important result vis a vis validation is that documents that were highly probable under Topic 5 (Table 1) were those with the highest average cosine similarity to the definition of a brick. That Topic 5 gives high probability to the terms build and house (see Table 1). Importantly, those terms do not appear in LSA comparison definition, so the fact that high probability documents under this topic were, on average, relatively similar to the dictionary definition cannot be attributed simply to a term-match. That is, in the topic model, a document reflecting this topic should be one that contains responses close to the normal use of bricks (i.e., building and in houses), and in LSA space, these documents, 
were indeed the most similar to the composite dictionary definition. This result provides preliminary validation of the current topic model as an adequate representation of the conceptual knowledge used by humans when generating creative uses for bricks.

\section{Discussion}

The goal of this paper was to provide a proof-of-concept that topic models, using LDA and Gibbs sampling, could adequately reflect the knowledge structure underpinning the AUT-brick responding process. A 15-topic model provided a valid reflection of knowledge in two ways. First, the most frequent topics were those that seemed to represent easily usable features of bricks (topics 2,4 , and 5), illustrating that like other creative thinking problems [18], there is a bias toward known uses of bricks (building), or exploitation of concrete properties such as the weight of the brick itself (e.g., weapon, paper weight). Second, the higher the frequency of the topic in the corpus, the more similar was that topic to the dictionary definition of a brick. This is validation of the model because the task explicitly involves a single category, and though participants are asked to generate alternative uses for the object in the category, it is sensible that most of the responses should be at least similar to the dictionary definition of a brick. Indeed, the 15-topic model yielded a topic distribution that correlated significantly with the average amount of semantic similarity (in LSA space) between a document from each topic and the dictionary definition.

Given these results, how useful is this model? First, the results are relevant to cognitive systems research in two ways. From a cognitive enhancement perspective, the topic model revealed the inherent bias in divergent thinking toward conventional uses for objects. As such, cognitive systems can be built to help humans overcome such biases in any creative thinking problem for which there exists a sufficient corpus. In this case, to aid humans in generating uses for bricks, a system could be designed that uses the topic and term distributions from normative data to generate suggestions as humans complete such a task. Since topics themselves can be thought of as a set of features [5], it may be relatively straightforward to design a cognitive system that samples lower probability features from an existing topic list, to provide simple prompts (likely verbs) to humans that might push their thoughts in a more unexpected or uncommon dimension. Second, the current model has implications for scoring AUT tasks, and providing automated feedback to humans. One common scoring system is the "flexibility" system, in which the frequency of category switches is tallied per response array per person. The system is relatively inefficient as it requires human hand-coding of datasets, and there seems to be no common system or set of norms with which this can be done. Griffiths and Steyvers [4] illustrated how terms in a document can be "tagged" using the term distribution. That is, within a document, the topic giving highest probability to each term can be tallied. Flexibility of divergent thinking responses is seen as a key measure of 
remote association such that the more flexible a set of responses is (i.e., the more switches) the more remote are the associations contained within.

In terms of application to the cognitive science of creativity, topic models such as this offer a structural explanation for the content generated during alternative uses responses. The data presented currently may not seem totally useful at first, as the model was only trained using responses from a single task. However, topic models need not be confined to a corpus from a single task (i.e., object), and future work in this area might focus on extracting a more abstracted set of topics that appear across alternative uses data from many different prompt objects. Several sources [3-5] provide details about how various ranges of hyperparameters for modeling topics with LDA influence the granularity of the resulting model. The parameters used presently are biased toward a more granular representation, which is appropriate in a corpus containing relatively homogeneous content. However, different values of $\alpha$ and $\beta$ specified when modeling a larger corpus can be used to extract more abstract topics. This would help cognitive models of creative thinking such that broader regularities in the kinds of features that humans seem to exploit while generating uses for objects can be examined. For example, modeling the topics across documents from different objects (i.e., tasks) can reveal whether different objects with different affordances lead to variation in the degree of remote association and use generation humans demonstrate. Arching back to the two explanations for divergent thinking described in the introduction, if it is the case that no matter which objects form the basis of the corpus, the regularities in responding are the same (i.e., bias toward concrete, conventional uses and features) it may mean that divergent thinking is distinguished from memory search, in general, by the iterative nature with which remote association must operate. However, if different objects lead to very different topic distributions-that some objects do not lead to many conventional terms in the term and topic distributions-it may be evidence that the search process operating during divergent thinking is not very different than that of normal memory, but that the particular constraints imposed by human understanding of the object constrain the search. Either way, topic models offer an exciting vehicle for further theoretical developments in divergent thinking, and creative thinking writ large.

\section{References}

1. Beaty, R. E., Silvia, P. J.: Why do ideas get more creative across time? An executive interpretation of the serial order effect in divergent thinking tasks. Psychology of Aesthetics, Creativity, and the Arts, 6(4), 309-319 (2012)

2. Beaty, R. E., Kenett, Y. N., Christensen, A. P., Rosenberg, et al.: Robust prediction of individual creative ability from brain functional connectivity. PNAS 201713532 (2018)

3. Blei, D., Ng, A., Jordan, M.: Latent Dirichlet allocation. JMLR 3, 993-1022 (2003)

4. Griffiths, T. L., Steyvers, M.: Finding scientific topics. PNAS 101, 5228-5235 (2004)

5. Griffiths, T. L., Steyvers, M., Tenenbaum, J. B.: Topics in semantic representation. Psychological Review, 114(2), 211-244 (2007) 
6. Hass, R. W.: Tracking the dynamics of divergent thinking via semantic distance: Analytic methods and theoretical implications. Memory \& Cognition, 45(2), 233244 (2017)

7. Hass, R. W.: Semantic search during divergent thinking. Cognition 166, 344-357 (2017)

8. Guilford, J. P.: The nature of human intelligence. McGraw-Hill, New York (1967)

9. Kenett, Y. N., Anaki, D., Faust, M.: Investigating the structure of semantic networks in low and high creative persons. Frontiers in Human Neuroscience 8(246), 407 (2014)

10. Landauer, T. K., Dumais, S. T.: A solution to Plato's problem: The latent semantic analysis theory of acquisition, induction, and representation of knowledge. Psychological Review 104(2), 211-240 (1997)

11. Mei, M., Minai, A. A.: Divergent thinking in a neurodynamical model of ideation. Neural Networks(IJCNN), 1825-1832 (2016)

12. Nusbaum, E. C., Silvia, P. J.: Are intelligence and creativity really so different? Intelligence 39(1), 36-45 (2011)

13. Olte?eanu, A.-M., Falomir, Z.: Object replacement and object composition in a creative cognitive system. Towards a computational solver of the Alternative Uses Test. Cognitive Systems Research, 39 15-32 (2016)

14. Raaijmakers, J. G., Shiffrin, R. M.: Search of associative memory. Psychological Review, 88(2), 93-134 (1981)

15. Feinerer, I., Hornik, K., Meyer, D.: Text Mining Infrastructure in R. Journal of Statistical Software 25(5) 1-54 URL: http://www.jstatsoft.org/v25/i05/ (2008)

16. Grun, B., Hornik, K.: topicmodels: An R Package for Fitting Topic Models. Journal of Statistical Software 40(13), 1-30. URL: http://doi.org/10.18637/jss.v040.i13 (2011)

17. Tversky, A.: Features of similarity. Psychological review, 84(4) 327-352 (1977)

18. Ward, T. B.: The role of domain knowledge in creative generation. Learning and Individual Differences 18(4), 363-366 (2008)

19. Wixted, J. T., Rohrer, D.: Analyzing the dynamics of free recall: An integrative review of the empirical literature. Psychonomic Bulletin \& Review, 1(1), 89-106 (1994) 